\title{
Une méthode d'étude des excavations souterraines en milieu viscoplastique. Prise en compte d'un état stationnaire des contraintes
}

\author{
A framework for the analysis of underground excavations \\ in viscoplastic medium, on account of a steady stress state
}

\author{
D. NGUYEN-MINH, A. POUYA \\ Laboratoire de Mécanique des Solides \\ (Laboratoire commun EP - EMSMP - CNRS URA 317)"
}

Rev. Franç. Géotech. n 59, pp. 5-14 (avril 1992)

\section{Résumé}

L'analyse de la convergence des galeries souterraines dans des milieux très fluants et pratiquement sans cohésion, tels que le sel gemme, certaines argiles molles, des sols gelés,... se heurte à des difficultés propres à ces matériaux, à savoir, la forte non linéarité du comportement et de la modélisation incertaine de la réponse à long terme à partir des essais de laboratoire, difficultés qui sont aggravées par la dispersion des caractéristiques mécaniques.

On propose pour cela un cadre d'étude comportant le choix d'une classe de lois de comportement représentatives des principales écoles de pensée, des hypothèses simplificatrices et une analyse adimensionnelle, de façon à faire une étude paramétrique du problème à partir d'un minimum de calculs.

L'existence d'un état de contrainte asymptotique pour ce type de problème, qui est une propriété remarquable, a été prise en compte.

Pour illustrer la méthode, on traite le cas des galeries et des cavités sphériques profondes isolées, et on propose des abaques.

\section{Abstract}

Analysis of underground galleries' closure in cohesionless creeping materials, such as rocksalt, soft clays, frozen soils,... is heavily impeded by the specific nature of these materials : they exhibit a large scattering in their mechanical characteristics, all the more their behavior is highly non linear, and the extension of the laboratory test results to long term periods is uncertain.

An appropriate method is proposed, on the basis of the choice of a class of viscoplatic flow laws supposed to be representative of the main different schools of thought, of simplifying hypotheses and of adimensional analysis, so that a parametric study of the problem is possible using a limited number of calculations.

Existence of a stationary stress state for such problems, which is an interesting feature, has been considered.

As an illustration, we study the case of a deep single circular gallery or spherical cavity, for which charts are calculated. 


\section{INTRODUCTION}

Les excavations profondes rencontrées en géotechnique (typiquement de 500 à plus de $1000 \mathrm{~m}$ ), dans des matériaux favorables au stockage (hydrocarbures. déchets...), ou à l'exploitation minière, subissent parfois des déformations différées importantes, qui exigent une étude particulière de l'évolution à long terme de ces structures. C'est le cas des matériaux tendres et fluants, tels que certaines évaporites (sel gemme, potasse...). des argiles molles et éventuellement des sols gelés.

Ainsi, la plupart des spécialistes s'accordent à attribuer à de tels matériaux une cohésion faible, voire nulle, et un comportement visqueux non linéaire. De tels matériaux ne rentrent pas strictement dans le cadre envisagé dans (NGUYEN-MINH, 1986).

La prévision à long terme de l'évolution des structures souterraines est cependant lourdement pénalisée par les particularités physiques du milieu telles que la dispersion des caractéristiques mécaniques, la forte non linéarité du comportement, la multiplicité des modèles phénoménologiques décrivant celui-ci, qui reflète, entre autres, la difficulté d'extrapolation dans le temps des lois expérimentales...

La dispersion des caractéristiques mécaniques dans le cas d'un sel de Bresse par exemple, est illustrée sur la figure 1 par le faisceau des courbes de fluage monoaxial alors que toutes les éprouvettes, provenant d'un sondage dans un même horizon, étaient soumises à des conditions de charge et de température identiques. Avec l'aide de minéralogistes du Laboratoire de Géologie du Muséum National d'Histoire Naturelle (CURIAL et MORETTO), la réponse en fluage des échantillons a d'ailleurs pu être corrélée à des faciès pétrographiques nettement différenciés, se rattachant, avec divers degrés d'impuretés, au mode de genèse du matériau. In situ, on pourrait ainsi s'attendre à un certain "effet d'échelle », fonction de la répartition géographique des différents faciès.

Pour l'analyse complète de l'évolution d'une structure donnée, il paraît ainsi tout à fait souhaitable d'effec-

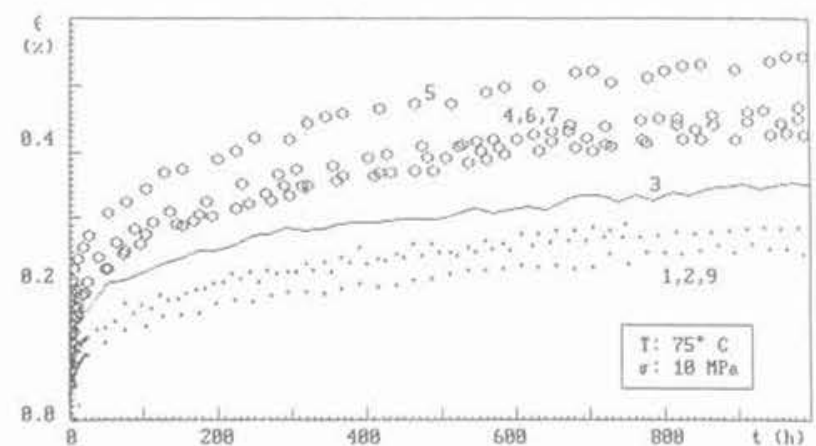

Fig. 1. - Effet de la dispersion sur la réponse en fluage monoaxial d'un sel de Bresse (France) , relation avec les faciès pétrographiques: phénoblastique $(1,2,9)$; laiteux $(4,5,6,7)$; catégorie intermédiaire (3).

Fig. 1. - Scattering of uniaxial creep response

of Bresse Salt (France) and relationship with petrographic variety of the samples: phenobastic $(1,2,9)$; milky $(4,5,6,7)$ intermediate category (3). tuer une étude paramétrique, qui, sous peine de ne pouvoir être économiquement réalisable, requiert la mise en ceuvre d'une méthodologie originale.

Dans ce but, en laissant de côté pour l'instant les phénomènes de rupture, on propose une approche générale, fondée :

a. sur le choix d'une classe de lois de comportement simples, mais suffisamment représentatives des différentes options rhéologiques pour le matériau;

b. sur des hypothèses simplificatrices pour le massif et son chargement ;

c. sur le traitement du problème en terme de paramètres adimensionnels.

Cette approche permet ainsi d'appréhender la généralité du problème, à partir d'un minimum de résultats de calcul, et de le présenter, entre autres, sous forme d'abaques susceptibles de couvrir un large spectre d'application.

A titre d'illustration de la méthode, on présente l'étude de la galerie horizontale profonde, en milieu isolé ; l'objectif étant d'étudier le comportement à long terme, les phases d'excavation ne sont pas prises en compte, et on supposera que le creusement est instantané. Le massif est pesant et soumis à une contrainte initiale hydrostatique. La cavité sphérique est également traitée.

\section{CHOIX DES LOIS DE COMPORTEMENT VISCOPLASTIQUE}

On admet la partition classique de la déformation totale $\epsilon$ en une déformation élastique $\epsilon^{\text {el }}$, supposée linéaire ( $\mathrm{E}$, module d'Young, et $\nu$, coefficient de Poisson) et une déformation différée $\epsilon^{\mathrm{vp}}$.

Les lois phénoménologiques, proposées dans la littérature pour décrire le comportement différé du sel gemme, peuvent être classées selon deux aspects : l'extrapolation dans le ternps des lois expérimentales de fluage unidimensionnel et leur généralisation tridimensionnelle.

\subsection{Extrapolation à long terme des lois de fluage}

Deux écoles de pensée s'opposent sur l'existence d'un régime de fluage secondaire. On a choisi parmi les lois les plus usuelles, qu'on a représentées avec leur dépendance éventuelle en température, dans la formule (1) : NORTON-HOFF (ou NORTON BAILEY) pour la première école, et LEMAÎTRE-MENZELSCHREINER pour la seconde. Un tel choix a été motivé d'ailleurs par des raisons pratiques, car ces lois, relativement simples, appartiennent à une même famille (NORTON: $\alpha=1$ ).

La première école, qui suppose l'existence du fluage secondaire, trouve sa justification dans des réflexions théoriques sur les mécanismes de déformation à l'échelle microphysique (LANGER et al., 1984; MUNSON et al., 1989...). Cette loi peut être complétée par la prise en compte d'une déformation de fluage primaire, en général une exponentielle amortie du temps, mais ceci ne sera pas considéré dans le cadre de cet article. 
Une des raisons évoquées par la seconde école (MENZEL et al., 1974 ; VOUILLE et al., 1983...) est que les essais de laboratoire, probablement du fait de leur durée limitée (de l'ordre du mois), ne mettent pas clairement en évidence une phase stationnaire. Cette deuxième loi est d'ailleurs classiquement utilisée pour les métaux en tant que loi de fluage primaire, sous le nom de loi de COTTRELL-ANDRADE (1910).

Du point de vue du dimensionnement, comme le suggère la figure 2, on pourrait considérer qu'à long terme, la loi de NORTOH-HOFF est plus pessimiste pour l'estimation des convergences de la structure, alors que la loi de LEMAITTRE-MENZEL-SCHREINER doit pouvoir donner des résultats plus précis à plus court terme, dans la mesure où elle rend bien compte de la phase de fluage primaire.

Lois unidimensionnelles retenues en fluage (1)

$\epsilon^{\mathrm{vp}}=\mathrm{a} \sigma^{\mathrm{n}} \mathrm{t}^{\alpha}$ déformation viscoplastique

$\alpha=1: \quad$ loi de Norton-Hoff

$\alpha \in$ ] 0,1 [ : loi de Lemaître-Menzel-Schreiner et

$\sigma$ contrainte axiale rapportée à $1 \mathrm{Mpa}$

$\begin{array}{ll}t & \text { temps en jour }(j) \\ a & \text { est en } j^{-\alpha} \text { avec : }\end{array}$

$\mathrm{a}=\mathrm{a}_{0} \exp (-\mathrm{K} / \mathrm{T})$

$\mathrm{K}$ constante d'activation thermique et $\mathrm{T}$ température absolue, en Kelvin

\subsection{Ecriture des lois de comportement tridimensionnelles}

La forme explicite en temps n'est valable que dans le cadre de l'essai de fluage unidimensionnel où l'état de contrainte est constant, bien que certains auteurs l'aient appliqué au calcul de structures (BORESI et al., 1982 ; LADANYI, 1974...). Lorsqu'il y a redistribution des contraintes, lapproximation précédente est cependant trop grossière, et il faut considérer la loi sous forme intrinsèque obtenue par élimination du temps; dans le cas où $\alpha<1$ (LEMAÎTRE-MENZEL-
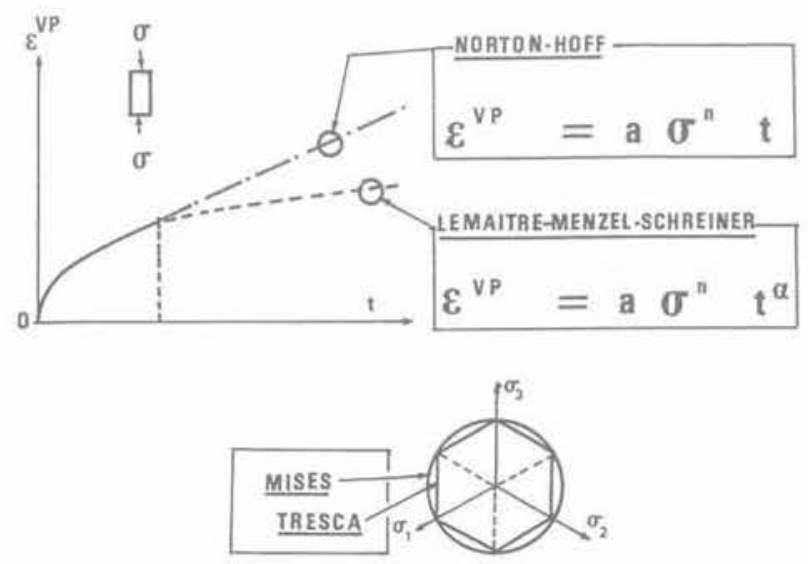

Fig. 2. - Choix des modèles de comporternent viscoplastiques. Fig. 2. - Choice of the viscoplastic models.
SCHREINER), on parle alors d'écrouissage en déformation, par opposition à l'écrouissage en temps du cas précédent.

La formulation tridimensionnelle de la loi, de type associé, fait intervenir en principe les trois invariants des contraintes ; le comportement anélastique de ces matériaux étant en général supposé purement déviatorique, on se limitera ici à l'utilisation des deux critères d'écoulement usuels: Mises ou Tresca.

Le critère de MISES est le plus utilisé pour le sel, mais il faut garder le second à l'esprit, d'autant plus qu'il conduit, pour la galerie, à des résultats sensiblement différents, et donnerait, selon certains auteurs, une meilleure interprétation de mesures in situ (MUNSON et al., 1989...).

La forme générale adoptée pour les lois est rappelée dans la formule (2).

$$
\text { Forme générale des lois }
$$

\section{NORTHON-HOFF :}

$\dot{\epsilon}^{\mathrm{vp}}=a f^{\mathrm{n}} \frac{\frac{\partial \mathrm{f}}{\partial \sigma}}{\left\|\frac{\partial f}{\partial \sigma}\right\|}$ où \|\| représente une norme

\section{LEMAÎTRE-MENZEL-SCHREINER :}

$$
\dot{\epsilon}^{\mathrm{vp}}=\frac{\partial}{\partial \mathrm{t}}\left(\xi^{\alpha}\right) \frac{\frac{\partial \mathrm{f}}{\partial \sigma}}{\left\|\frac{\partial \mathrm{f}}{\partial \sigma}\right\|} ; \quad \xi=[\mathrm{a} \mathrm{f}]^{1 / \alpha}
$$

La dépendance éventuelle de a en température est donnée en (1).

Les définitions de la fonction de charge $\mathrm{f}$ et de la norme || || dépendent de la loi d'écoulement choisie :

1. MISES : $\mathrm{f} \equiv \sqrt{3 \mathrm{~J}_{2}}$

$\mathrm{J}_{2}=\frac{1}{2} \operatorname{tr}\left(\mathrm{s}^{2}\right) ; \mathrm{s}$ déviateur des contraintes ;

$$
\|s\|=\sqrt{\frac{2}{3} \operatorname{tr}\left(s^{2}\right)}
$$

2. TRESCA : $f \equiv\left(\sigma_{1}-\sigma_{3}\right)$

$\sigma_{1}>\sigma_{2}>\sigma_{3}$ contraintes principales ordonnées;

$$
\|\sigma\|=\frac{1}{2}\left(\left|\sigma_{1}\right|+\left|\sigma_{2}\right|+\left|\sigma_{3}\right|\right)
$$

Il faut distinguer ici :

- le régime face, où $\sigma_{2}$ est strictement intermédiaire, alors :

$$
\dot{\epsilon}_{2}^{\mathrm{vp}}=0 \text { car } \frac{\partial \mathrm{f}}{\partial \sigma_{2}}=0
$$

- le régime d'arête lorsque $\sigma_{2}=\sigma_{1}$ par exemple, $\mathrm{s}$ : alors

$$
\dot{\epsilon}_{2}^{\mathrm{vp}} \neq 0
$$




\section{CONSÉQUENCE RÉSULTANT DES LOIS DE COMPORTEMENT : EXISTENCE D'UN ÉTAT DE CONTRAINTE ASYMPTOTIQUE}

Sous l'effet d'un chargement extérieur fixe, les cavités souterraines étudiées dans le cadre de cette étude subissent, après creusement, un régime transitoire impliquant une redistribution des contraintes dans le massif ; dans certains cas, le champ de contrainte peut tendre vers un état asymptotique, en parfaite analogie avec celui d'une éprouvette en fluage.

Dans le cas des lois de fluage secondaire (NORTONHOFF en particulier), plusieurs auteurs ont remarqué et exploité pour le calcul des structures, l'existence de cet état asymptotique : ADQVIST et HULT (1962), HULT (1964),... Ainsi MANDEL (1960) démontre un théorème pour les structures soumises à un "fluage » ou à une " relaxation ", pour une classe de matériaux dits de "Maxwell généralisés ", définis par :

$\dot{\epsilon}=\dot{\epsilon}^{e l}+\dot{\epsilon}^{v p}$

$\dot{\epsilon}^{e l}=\partial / \partial t\left\{\partial \Phi_{c} / \partial \sigma\right\}$ vitesse de déformation élastique $\dot{\epsilon}^{\mathrm{vp}}=\partial \Psi_{\sigma} / \partial_{\sigma}$ vitesse de déformation viscoplastique $\Psi_{c} \quad$ potentiel complémentaire de dissipation

Une telle formulation englobe la loi de NORTONHOFF, celles de PRANDTL-NADAI, EYRINGSODERBERG... ainsi que des lois à seuil, comme celle de BINGHAM. Un exemple trivial d'état asymptotique est fourni par les modèles linéaires de MAXWELL et KELVIN, pour lesquels l'état de contraintes asymptotique est atteint instantanément et n'est autre le champ de contrainte élastique initial.

Par contre, il n'existe pas à notre connaissance, de théorème équivalent pour la viscoplasticité avec écrouissage. En suivant une démarche similaire à celle de MANDEL, nous avons pu étendre ce résultat aux lois suivantes, dont la forme générale contient le modèle de LEMAîTRE-MENZEL-SCHEINER, ainsi que celui de MUNSON et al. (1989)... (la démonstration de théorème n'est pas donnée dans cet article) :

$$
\dot{\epsilon}^{\mathrm{vP}}=\mathrm{G}(\chi) \partial \Psi_{\mathrm{c}} / \partial \sigma \quad \mathrm{G}, \Psi_{\mathrm{c}} \geq 0
$$

avec :

$$
\chi=\int_{0}^{t}\left\|\epsilon^{\mathrm{vp}}\right\| \mathrm{dt}
$$

et : $\quad \mathrm{dG} / \mathrm{d} \chi<0$ (fluage de type primaire)

L'état asymptotique des contraintes, définie par $\dot{\sigma}=0$., soit $\epsilon^{e l}=0$, jouit de certaines propriétés intéressantes :

a. cet état ne dépend pas des propriétés élastiques, puisque dans ce cas la vitesse de la déformation élastique, proportionnelle à $\sigma$ est nulle (ceci reste valable dans le cas plus général où la déformation élastique est non linéaire mais dérive d'un potentiel) ; b. l'état asymptotique des contraintes est le même pour NORTON-HOFF et LEMAÎTRE-MENZELSCHREINER, pour un paramètre $\mathrm{n}$ donné. La fonction de charge $f$ (voir formule 2), tendant avec les contraintes vers des valeurs fixes, le champ des déformations est alors donné par:

$$
\frac{\partial \epsilon}{\partial t^{\alpha}}=a f^{n}\left[\frac{\partial f}{\partial \sigma}\right] /\left[\left\|\frac{\partial f}{\partial \sigma}\right\|\right]
$$

qui montre, après utilisation des relations de compatibilité des déformations, que le champ de contraintes aysmptotique est indépendant de $\alpha$;

c. l'état asymptotique fournit un repère précieux pour les calculs numériques en régime transitoire.

\section{CAS DE GALERIES HORIZONTALES ET PARALLÈLES : HYPOTHÈSES DE DÉFORMATION PLANE ET D'INCOMPRESSIBILITÉ}

Nous nous plaçons désormais dans le cadre de la déformation plane, applicable à l'étude de tunnels horizontaux suffisamment longs et parallèles, creusés dans le massif pesant, soumis à un état initial de contrainte hydrostatique.

Nous faisons l'hypothèse d'incompressibilité pour le massif, qui revient à prendre dans ce cas un coefficient de Poisson $\nu=0,5$; compte tenu de la valeur habituelle de ce coefficient, de l'ordre de 0,25, on vérifie, dans le cadre du problème traité, que cette hypothèse ne conduit dans les cas les plus défavorables, qu'à des écarts relatifs inférieurs à $10 \%$ sur la convergence de la galerie, ce qui est tout à fait acceptable dans le cadre de notre démarche. Par contre il résulte des avantages importants.

\subsection{Simplifications de l'écriture des lois}

- Il n'y a pas de régime d'arête pour le critère de Tresca, la contrainte $\sigma_{2}$ normale au plan de la déformation étant strictement intermédiaire, car $\sigma_{z}=1 / 2$ $\left(\sigma_{1}+\sigma_{3}\right)$

- pour la même raison, pour MISES, $\frac{\partial f}{\partial \sigma_{2}} \equiv 0$ : la la déformation viscoplastique $\epsilon_{\mathrm{s}}^{\mathrm{vp}}$ rest nulle, comme pour TRESCA.

Les critères de TRESCA et MISES sont donc identiques à une constante de proportionalité près, ce qui permet d'écrire les lois sous un même formalisme, le passage de l'une à l'autre se faisant par un simple changement de constante :

$$
\begin{aligned}
& \partial / \partial \mathrm{t}\left(\epsilon^{\mathrm{vp}}\right)=\partial / \partial \mathrm{t}\left(\xi^{\alpha}\right) \partial \mathrm{f} / \partial \sigma ; \quad \dot{\xi}=\left\{\mathrm{a}_{\mathrm{r}} \mathrm{f}^{\mathrm{n}}\right\}^{1 / \alpha} \\
& \mathrm{f} \equiv \sigma_{1}-\sigma_{3} ; \sigma_{1}>\sigma_{3} \text { et } \sigma_{2}=\sigma_{\mathrm{z}}=\frac{\sigma_{1}+\sigma_{3}}{2} \\
& \alpha=1: \quad \text { Norton-Hoff } \\
& \mathrm{a}_{\mathrm{r}}=\mathrm{a} \rightarrow \text { Tresca } ; \quad \mathrm{a}_{\mathrm{r}}=\mathrm{a}(\sqrt{3} / 2)^{\mathrm{n}+1} \rightarrow \text { Mises }
\end{aligned}
$$




\subsection{Transformation du problème avec pesanteur en un problème sans pesanteur}

L'incompressibilité du matériau entraîne dans ce cas, un comportement purement déviatorique ce qui permet, compte tenu de l'hypothèse sur l'état initial de contrainte hydrostatique, de se ramener au moyen d'un changement de variable sur les contraintes, (MANDEL, 1976), à un problème non pesant, en remplaçant le gradient de la pesanteur par une pression uniforme appliquée en surface (fig. 3). Le creusement de la galerie se traduit alors par la décroissance de la pression $\mathrm{P}_{\mathrm{i}}$ en paroi suivant un certain historique.

Cette remarque vaut pour une structure souterraine quelconque : galerie de section de forme quelconque, système de galeries situées à des profondeurs équivalentes. Il est à noter que les puits verticaux n'entrent pas, en toute rigueur, dans le cadre ainsi défini.

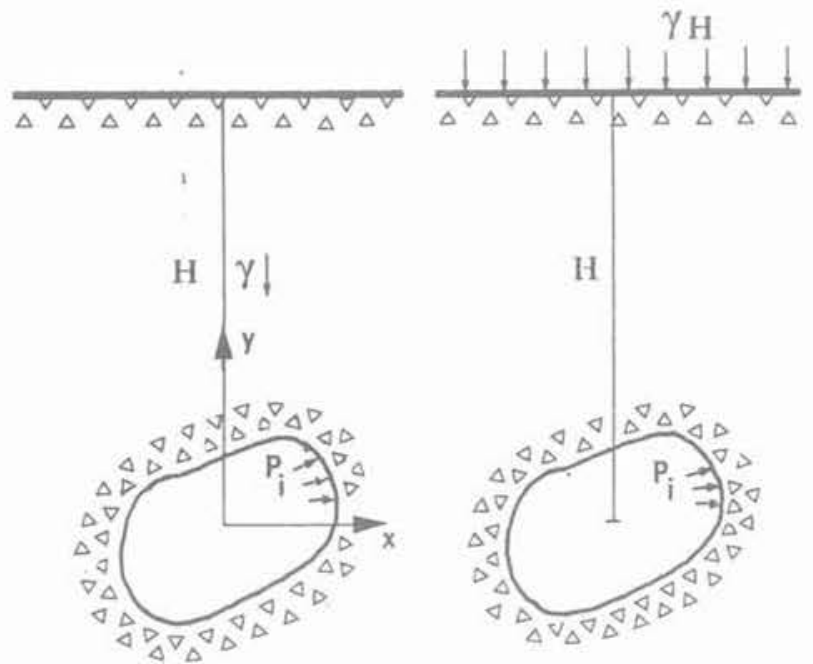

Fig. 3. - Transformation du problème pesant et non pesant. Fig. 3. - Transformation of the problem with gravity into a one without gravity.

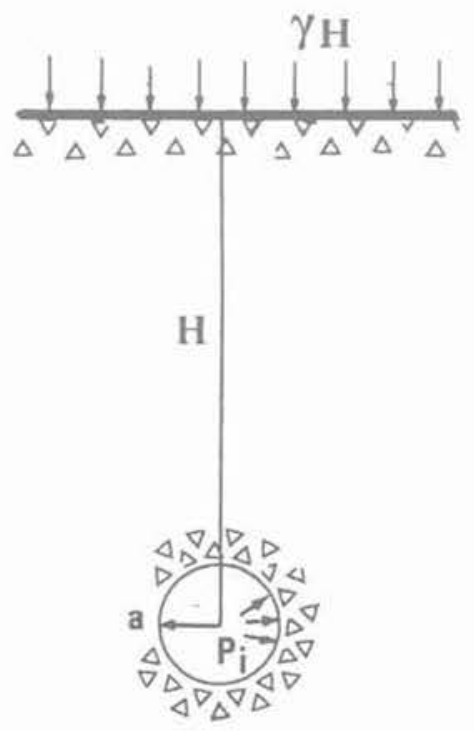

\section{5. ÉCRITURE DES ÉQUATIONS DU PROBLÈME \\ EN VARIABLES ADIMENSIONNELLES}

Nous allons adimensionnaliser les variables du problème en effectuant sur les contraintes, les déformations et le temps, un changement d'échelle par rapport aux paramètres caractéristiques $\sigma_{0}, \epsilon_{0}, \tau_{0}$, de façon à obtenir des équations de comportement indépendantes des paramètres mécaniques du problème:

$\sigma \rightarrow \sigma^{*}=\sigma / \sigma_{0} \quad \epsilon \rightarrow \epsilon^{*}=\epsilon / \epsilon_{0} \quad \tau \rightarrow \mathrm{t}^{*}=\mathrm{t} / \tau_{0}$ avec :

$\sigma_{0}$ contrainte positive, choisie proportionnelle au chargement $\left(\mathrm{P}_{\mathrm{e}}-\mathrm{P}_{\mathrm{f}}\right)$,

$\epsilon_{0}=\sigma_{0} / E^{\prime}=3 / 4 \sigma_{0} / E$ et $\tau_{0}=\left(1 / a_{x} \epsilon_{0} \sigma_{0}^{-n}\right)^{1 / \alpha}$ $\sigma_{0}$ et $\epsilon_{0}$ sont ainsi des quantités reliées au comportement élastique de la structure.

\section{EXEMPLE D'APPLICATION : CONVERGENCE LIBRE D'UNE GALERIE ISOLÉE CIRCULAIRE HORIZONTALE}

On a analysé les divers choix permettant d'élaborer un cadre général d'étude visant à décrire, compte tenu des diverses incertitudes, le comportement d'une structure souterraine dans un massif salifère, à partir d'un minimum de calculs.

A titre d'exemple, on présente le cas de la galerie horizontale circulaire isolée à grande profondeur. Si on admet que l'état de contrainte * au loin * dans le problème transformé peut être considéré comme uniforme, et égal à celui de l'état initial (pression $\left.\mathrm{P}_{\mathrm{e}}=\gamma \mathrm{H}\right)$, on peut admettre lhypothèse de la symétrie circulaire (fig. 4). Par la suite, la pression $\mathrm{P}_{e}$ et le rayon extérieur $\rho$ du tube seront indépendants.

Fig. 4. - Hypothèse de la symétrie circulaire pour la galerie profonde isolée.

Fig. 4. - Circular symetry hypothesis for the deep single gallery. 
Comme on se propose d'étudier ici le comportement à long terme de la galerie, on négligera les phases du creusement : on considère donc le fluage libre de la galerie sous l'action d'une annulation brutale de la pression intérieure $\mathrm{P}_{\mathrm{i}}$.

Remarque : il conviendra d'observer une certaine prudence vis-à-vis de l'hypothèse de symétrie circulaire. puisqu'en toute rigueur, en viscoplasticité sans seuil, le principe de Saint-Venant ne s'applique plus et peut être mis en défaut dans certains cas limites.

\subsection{Equations générales}

$\mathrm{u}$ étant le déplacement radial, $\sigma_{\mathrm{r}}$ et $\sigma_{\theta}$ les contraintes principales radiales et circonférentielles, les équations générales du problème sont les suivantes:

$$
\begin{array}{ll}
\frac{\partial \sigma_{r}}{\partial_{r}}=\frac{\sigma_{\theta}-\sigma_{\mathrm{r}}}{r} & \text { équilibre } \\
\frac{\partial \mathrm{u}}{\partial \mathrm{r}}+\frac{\mathrm{u}}{\mathrm{r}}=0 & \text { incompressibilité } \\
\frac{\mathrm{u}}{\mathrm{r}}=\frac{\sigma_{\theta}-\sigma_{\mathrm{r}}}{\mathrm{E}^{\prime}}-\epsilon_{\mathrm{r}}^{\mathrm{vp}} & \mathrm{E}^{\prime}=4 / 3 \mathrm{E}
\end{array}
$$

où $\epsilon_{\mathrm{r}}^{\mathrm{vp}}$ est la déformation viscoplastique radiale, dont la vitesse est donnée par (4).

On choisit le paramètre caractéristique $\sigma_{0}$ comme étant le déviateur de Tresca élastique initial en paroi, sous l'effet de la chute instantanée de la pression intérieure de $\mathrm{P}_{e}$ à $\mathrm{P}_{i}$, soit :

$$
\sigma_{0}=2\left(\mathrm{P}_{e}-\mathrm{P}_{1}\right) /\left(1-\rho^{-2}\right)
$$

$\epsilon_{0}$ représente alors la convergence initiale correspondante, en valeur absolue.

\subsection{Phase transitoire}

L'évolution est solution d'un système d'équations intégro-différentielles fonction de la déformation viscoplastique radiale $\epsilon_{\mathrm{r}}^{\mathrm{vp}}$, et de la convergence en paroi $\mathrm{U}^{\prime}$, et nécessite une résolution numérique :

$$
\begin{aligned}
& \partial / \partial \mathrm{t}\left[\left(\epsilon_{\mathrm{r}}^{\mathrm{vp}}\right)^{1 / \alpha}\right]=\mathrm{f}^{\mathrm{n} / \alpha} \\
& \mathrm{f}=\left(\sigma_{\mathrm{r}}-\sigma_{\theta}\right)=\mathrm{U}_{\mathrm{i}} / \mathrm{r}^{2}+\epsilon_{\mathrm{r}}^{\mathrm{vp}} \\
& \mathrm{U}_{\mathrm{i}}=-1-2 /\left(1-\rho^{-2}\right) \int_{1}^{\rho} \frac{\epsilon_{\mathrm{r}}^{\mathrm{vp}}}{\mathrm{r}} \mathrm{dr} \\
& \text { avec } \epsilon_{\mathrm{r}}^{\mathrm{vp}} \equiv 0 \quad \text { pour } \mathrm{t}=0 .
\end{aligned}
$$

Toutefois, dans le cas du matériau de NORTON, on peut se ramener à l'équation intégro-différentielle pour la fonction de charge $f$ (déviateur de TRESCA):

$\partial \mathrm{f} / \partial \mathrm{t}+\mathrm{f}-2 /\left[\mathrm{r}^{2}\left(1-\rho^{-2}\right)\right] \int_{1}^{\rho} \frac{\mathrm{f}^{\mathrm{n}}}{\mathrm{r}} \mathrm{dr}=0$

dont une solution approchée explicite a pu être trouvée (Annexe 2).

La figure 5 montre, pour le milieu de NORTONHOFF, la comparaison très satisfaisante du déviateur

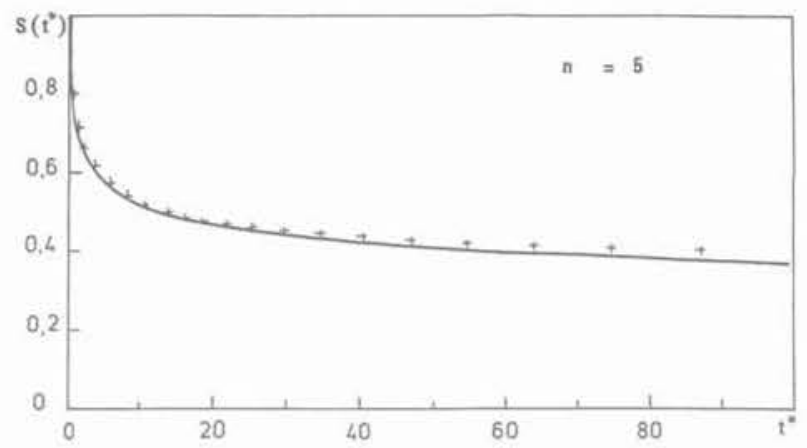

Fig. 5. - Comparaison du déviateur

à la paroi calculé numériquement (-) et par la solution explicite approchée $(+1$. (Loi de Norton-Hoff, $n=5$ ).

Fig. 5. - Comparison between the stress deviator at the wall obtained from numerical analysis (-) and from the approximative closed form solution $(+1$. (Norton-Hoff law, $n=5$ ).

en paroi obtenu numériquement et par la solution explicite approchée.

Remarque importante :

On peut établir l'équivalence formelle entre les calculs cylindrique et sphérique.

Avec la symétrie sphérique, on aboutit à un système d'équations comparable à (8) ; en fait, on montre que les deux systèmes d'équations sont identiques, après un changement de variable d'espace $\xi=r^{2}$ (cas cylindrique) et $\xi=r^{3}$ (cas sphérique); pour ce dernier, il convient de redéfinir les constantes mécaniques :

$$
\begin{aligned}
& E^{\prime \prime}=\mathrm{E} /(1-\nu)=2 \mathrm{E} ; \\
& \sigma_{0}=3 / 2\left(\mathrm{P}_{\mathrm{e}}-\mathrm{P}_{\mathrm{f}}\right) /\left(1-\rho^{-3}\right) ; \\
& \epsilon_{0}=\sigma_{0} / \mathrm{E}^{\prime \prime} \\
& \mathrm{a}_{r} \text { (Mises ou Tresca) }=\mathrm{a} / 2
\end{aligned}
$$

on notera bien entendu la correspondance géomérique : $\rho^{2}$ (cylindre) $=\rho^{3}$ (sphère)

Les résultats se transposent ainsi facilement d'un cas à l'autre. Il est à noter que cette propriété intéressante résulte de l'hypothèse d'incompressibilité.

\subsection{Etat asymptotique}

Celui-ci s'obtient en faisant $\dot{\sigma}=0$, ce qui donne, pour la convergence en paroi $U_{1}$ et le déviateur des contraintes :

$$
\begin{aligned}
& U_{i}=A^{n} t^{\alpha}+C^{t e} \\
& \sigma_{r}-\sigma_{\theta}=A r^{-2 / n} \\
& A=\frac{1}{n} \frac{1-\rho^{-2}}{1-\rho^{-2 / n}}
\end{aligned}
$$

On remarquera que la pente asymptotique $\mathrm{dU}_{\mathrm{t}} / \mathrm{dt}^{\alpha}$ $=A^{n}$ est très sensible à l'influence de la géométrie, représentée ici par le paramètre $\rho$. Ainsi, avec $n=5$, ce paramètre vaut $3,8510^{-3}$ pour $\rho=10$, alors qu'il vaut $5,510^{-4}$ pour $\rho=400$. 
Remarque : effet des déplacements finis :

Il convient de préciser que l'état asymptotique des contraintes est lié à l'hypothèse des petites transformations; un calcul élémentaire avec le modèle de NORTON-HOFF montre en fait qu'en tenant compte du changement de géométrie, les formules (10) deviennent :

$$
\mathrm{R}=\mathrm{R}_{0} \exp \left(-\mathrm{n}^{\mathrm{n}} \mathrm{t}\right) \quad \sigma_{\mathrm{x}}-\sigma_{\theta}=\frac{1}{\mathrm{n}}\left[\frac{\mathrm{R}}{\mathrm{r}}\right]^{2 / \mathrm{n}}
$$

où $\mathrm{R}$ est le rayon actuel de la galerie, $\mathrm{R}_{0}$ son rayon initial.

Si l'on se place en un point géométrique $r$ fixe, on verra ainsi décroître dans le temps le déviateur avec la fermeture de la galerie.

\section{RÉALISATION \\ ET UTILISATION DES ABAQUES}

\subsection{Abaques de convergence}

Le calcul de l'intégrale dans (8) nécessite la donnée du rayon $\rho$; or, on a vu que ce paramètre influait énormément sur la vitesse de convergence limite, à l'état asymptotique. Pour rendre compte d'un massif * infini *, il fallait prendre $\rho$ suffisamment grand ; en se limitant à $\rho=400$ on se donnait comme cas limite de référence une galerie de $3 \mathrm{~m}$ de rayon lou une cavité de $22 \mathrm{~m}$ de rayon) à $1200 \mathrm{~m}$ de profondeur. Les calculs ont été effectués sur un micro PC.

La figure 6 donne, pour diverses valeurs de $n$, les abaques de convergence en fonction du temps pour NORTON-HOFF en paramètres adimensionnels, en coordonnées bilogarithmiques.

Pour LEMAÎTRE-MENZEL-SCHREINER, on obtient des courbes presque superposables en prenant $t^{*} \alpha$ à la place de $\mathrm{t}^{\circ}$; la figure 7 donne, pour quelques valeurs de $\mathrm{n}$ et $\alpha$, la correction $\delta \mathrm{X}$ de translation suivant l'abscisse permettant de ramener, pour une valeur de $\mathrm{n}$ donnée, la courbe de convergence de LEMAITRE-MENZEL-SCHREINER sur celle de NORTON-HOFF. On voit, pour la plupart des cas, surtout si n est inférieur à 3 , que la correction $\delta \mathrm{X}$ reste modérée, et qu'on peut, en première approximation, se contenter des abaques pour NORTON$\mathrm{HOFF}$, en prenant $t^{\alpha}$ à la place de $t$.

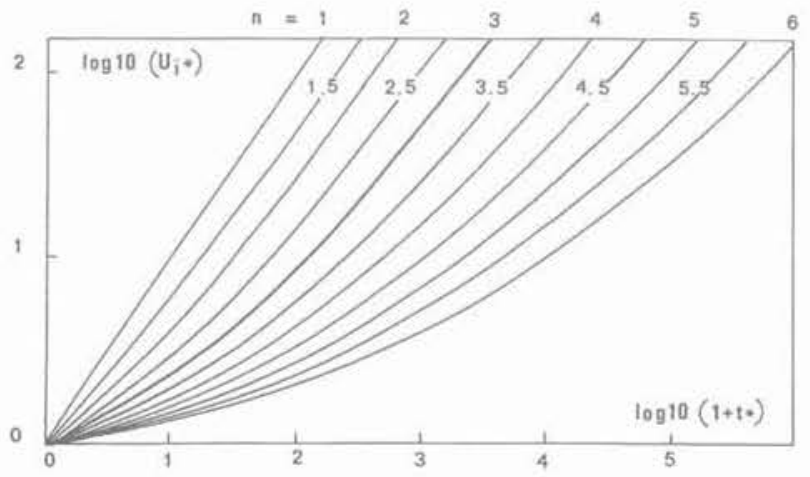

Fig. 6. - Abaques donnant la convergence en fonction du temps. Loi de Norton-Hoff.

Fig. 6. - Abacus for closure vs time. Norton-Hoff law.
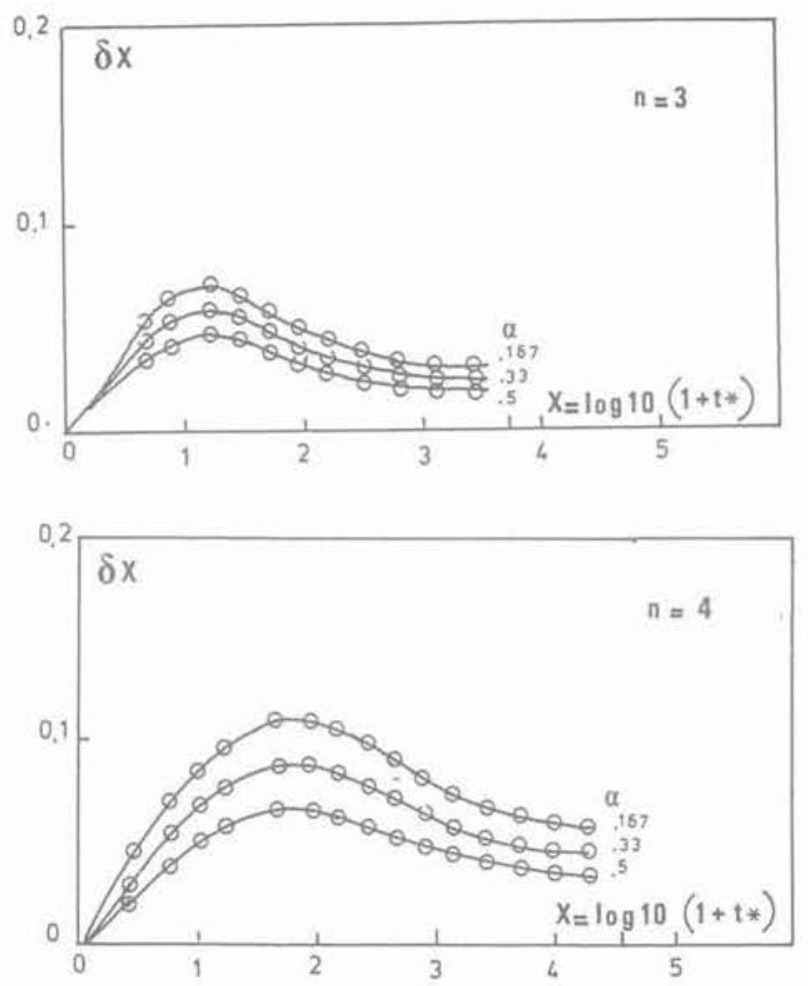

Fig. 7. - Corrections par translation selon l'abscisse pour ramener le cas de LEMAITRE-MENZEL-SCHREINER a celui de NORTON-HOFF $(n=3$ et 4$)$.

Fig. 7. - Translation correction parallel to the abscissa to convert LEMAITRE-MENZEL-SCHREINER case into NORTON-HOFF one $(n=3$ and 4$)$.

\subsection{Exemples d'utilisation des abaques} dans le cas du matériau de NORTON-HOFF

Soit par exemple, le cas d'une galerie qu'on veut creuser à $600 \mathrm{~m}$ de profondeur, correspondant, avec une densité de terrain de 2,2 , à une pression géostatique de 13,2 $\mathrm{MPa}$.

La température à cette profondeur est supposée être de l'ordre de $32,6^{\circ} \mathrm{C}$, correspondant à $15^{\circ} \mathrm{C}$ en surface et à un gradient géothermique de $1^{\circ} \mathrm{C}$ tous les $34 \mathrm{~m}$; le module d'Young est supposé être de $25000 \mathrm{MPa}$.

Si on admet pour le matériau de NORTON-HOFF les caractéristiques moyennes suivantes :

$$
\mathrm{K}=4755 \quad \mathrm{a}_{0}=0,0435 \mathrm{j}^{-1} \quad \mathrm{n}=3
$$

on obtient, en admettant un critère de Mises pour le calcul de $\tau_{0}$, les valeurs suivantes pour les paramètres caractéristiques :

$\sigma_{0} \approx 2 \mathrm{P}_{\mathrm{e}}=26,4 \mathrm{MPa}$

$\epsilon_{0}=\frac{3}{4} \frac{\sigma_{0}}{\mathrm{E}}=7,9210^{-4} \quad \tau_{0}=10,06 \mathrm{j}$

ce qui permet de convertir les diagrammes adimensionnels en diagrammes dimensionnels. La figure 8 montre ainsi les courbes de convergence \& moyennes , pour une certaine plage des valeurs de $n$ : 2,$5 ; 3 ; 3,5$. 


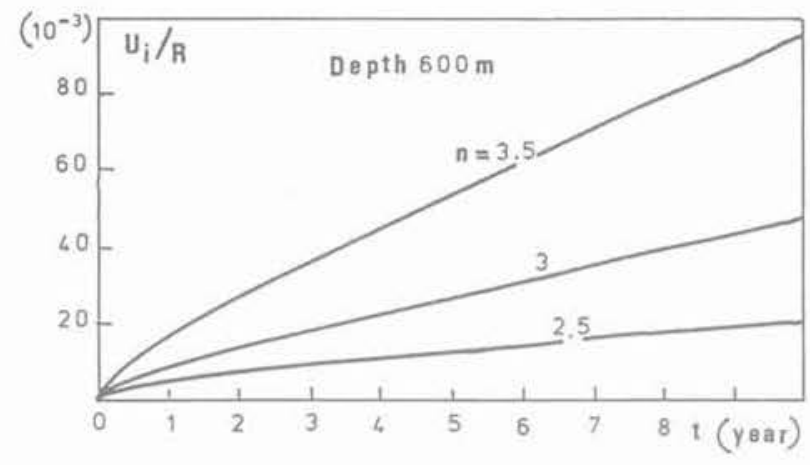

Fig. 8. - Convergence en fonction du temps à profondeur fixée, en coordonnées réelles (NORTON-HOFF).

Fig. 8. - Closure versus time for given depth in actual coordinates (NORTON-HOFF).

A partir d'une telle courbe de convergence " moyenne », (en tiretés, figure 9), on peut faire une étude de sensibilité du comportement lorsque varient les caractéristiques du matériau, n étant fixé. De l'expression des paramètres caractéristiques on déduit des transformations géométriques simples correspondant aux variations des constantes, ce qui permet ainsi une estimation graphique des variations. Par exemple:

a. si on change le module d'Young, la nouvelle courbe se déduit de la précédente, dans le cas du matériau de NORTON-HOFF, par une homothétie par rapport à l'origine de rapport inverse des modules E. Ainsi certains auteurs préconisent un module d'Young réduit pour tenir compte d'un fluage primaire ; cette transformation permet dans ce cas de connaître immédiatement le résultat d'une telle hypothèse :

b. si on fait varier les autres paramètres, en dehors de $E$, il y a modification des temps caractéristiques : la nouvelle courbe se déduit alors de la première par une affinité suivant l'axe des ordonnées de rapport inverse de celui des temps caractéristiques.

Notons que pour LEMAITTE-MENZEL-SCHREINER, les transformations géométriques précédentes sur les courbes de convergence en variables dimensionnelles, sont les mêmes que pour NORTON-HOFF à condition de se placer dans les axes $U_{1}, t^{\alpha}$.

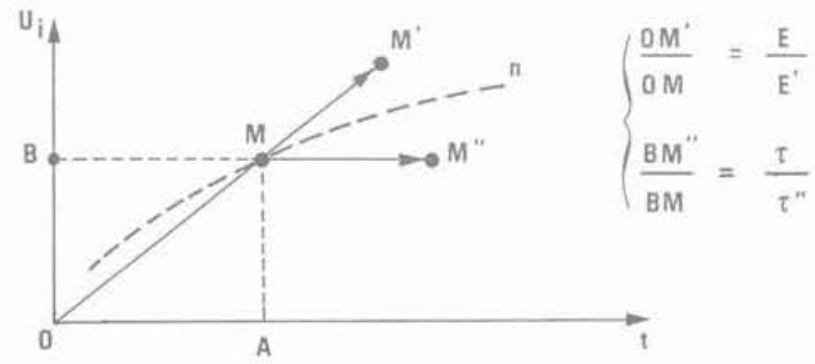

Fig. 9. - Transformation géométrique de la courbe de convergence (en coordonnées réelles) consécutive à un changement du module de YOUNG E ou du temps caractéristique $\pi$.

Fig. 9. - Geometrical transformation of the closure curve (in actual coordinates) due to a change of the YOUNG modulous. E or characteristic time $\tau$.
Les exemples étudiés privilégient l'étude de la convergence (resp. du déviateur en paroi), mais on peut envisager bien entendu d'autres formes d'exploitation des abaques, privilégiant par exemple l'étude de la profondeur ou du temps en fonction des autres paramètres.

\section{CONCLUSION}

On a illustré sur un cas particulier, une méthode permettant d'évaluer globalement le comportement des excavations dans le sel gemme, à partir d'un minimum de résultats obtenus suivant les principes généraux évoqués ci-dessus ; cette méthode permet entre autres, d'étudier facilement la sensibilité des résultats aux divers paramètres mécaniques, de comparer les différentes options rhéologiques et l'effet de la géométrie (cylindrique, sphérique), en utilisant de simples relations d'équivalence à partir d'une série d'abaques de base.

L'exploitation de ces résultats peut aussi se faire par un programme informatique, qui, par sa très grande rapidité d'exécution, se prête particulièrement à une étude paramétrique (logiciel CAVITUS).

D'autres applications ont été menées ou sont en cours, et requièrent dans le cas général le recours au calcul numérique par éléments finis (interaction de galeries, soutènement,...) ; elles montrent clairement l'intérêt de cette approche qui permet de tirer, au fur et à mesure des études effectuées, des lois de dimensionnement pour les structures dans le milieu concerné, dont la généralité transcende les incertitudes attachées à la dispersion des caractéristiques mécaniques ou même au caractère approché des lois.

\section{REMERCIEMENTS}

Cette étude a été financée en partie par l'ANDRA.

\section{BIBLIOGRAPHIE}

BEREST P., NGUYEN-MINH D. (1983), Deep underground storage cavities in rock salt: Interpretation of in situ data from french and foreign Sites. Proc. $1^{\text {st }}$ Conf. on the Mechanical Behavior of Salt. Pennsylvania State University. Nov. 1981, Trans. Tech. Pub., Clausthal, Germany.

BORESI A.P., DEERE D.U. (1963), Creep closure of a spherical cavity in an infinite medium (With special attention to Project Dribble, Tatum Salt Dome, Mississipi, for Holmes and Narver, Inc., Las Vegas Division.

BORM G. (1987), Bohrlochkonvergenz und Span. nungsrelaxation im Steinsalzgebirge. Proc. $6^{\text {th }}$ Cong. ISRM, Montreal, Canada.

HULT J. (1962), On the stationarity of stress and strain distributions in creep. Proc. IUTAM Symp. on Second Order Effects in Elasticity. Plasticity, and Fluid Dynamics, Haifa, Israël, 1962, (Eds Reiner \& Abir) pp. 352-361.

MANDEL J. (1960), Généralisation non linéaire des corps de Maxwell et de Kelvin. Fluage et relaxation. Colloque int. de Rhéologie. Pub. CNRS. 
MANDEL J. (1974 a), Plasticité et poussée des terres, science des matériaux. Fasc II. Cours ENSMP.

MANDEL J. (1974 b), Unicité et principes variationnels en viscoplasticité. Quelques résultats. Séminaire « Plasticité et viscoplasticité, 1972 ». Laboratoire de mécaniques des solides, Ecole Polytechnique, Paris. Ediscience/McGraw-Hill.

LADANYI B. (1972), An engineering theory of creep in frozen soils. Canadian Geotechnical Journal, 9, pp. 63-80.

LANGER M., WALLNER M, WASSMANN Th.H. (1984), Gebirgsmechanische Bearbeitung von Stabilitatsfragen bei Deponiekavernen im Salzgbirge. Kali u. Steinsalz 9, 2, S. 66-76, Essen 1984.

MUNSON D.E.. FOSSUM A.F. (1989), Approach to first model prediction of measured WIPP In situ room closure in salt. Proceedings 30 th US Symposium on Rock Mechanics. Univ. West Virginia University, Morgantown.

MENZEL W., SCHREINER W. (1977), Zum geomechanischen Verhalten von Steinsaitzverschiedenen Lagerstatten der DDR. Neue Bergbautechnik, vol. 7. Teil II, 565-571.

NGUYEN-MINH D. (1986), Modèles rhéologiques pour l'analyse du comportement différé des galeries profondes. Cong. Int. ITA/AITES: Grands ouvrages en souterrain, Florence, Italie, $\mathrm{pp}$. 659-666.

ODQVIST F.K.G, HULT J. (1962), Kriechfestigkeit metallischer Werstoffe. Springer Verlag. Berlin.

VOUILLE G., TIJANI S.M., de GRENIER F. (1984), Experimental determination of the rheological behaviour of Tersanne Rock Salt. Proceedings First Conference on the Mechanical Behavior of Salt, Pennsylvania State University, Novembre 1981, Trans. Tech. Publications, Clausthal, Germany, pp. 407-420.

\section{ANNEXE}

Convergence d'une galerie circulaire en massif infini de NORTON-HOFF : solution analytique approchée pour les contraintes

Nous considérons le cas du milieu infini $(\rho-\infty)$; reprenons l'équation (9) dans laquelle nous faisons le changement de variable:

$$
\zeta=\frac{1}{r^{2}}
$$

Notons par la suite $F(\zeta, t) \equiv f(r, t)$, et $\vec{F} \equiv \frac{\partial F}{\partial t}$; on obtient l'équation intégro-différentielle : avec la condition initiale:

$$
\mathrm{F}(\zeta, 0)=\zeta \quad \text { (élasticité instantanée) }
$$

Cette équation est également valable dans le cas sphérique en prenant $\zeta=\frac{1}{r^{3}}$.

On voit que $F$ vérifie:

(A.4) $\int_{0}^{1} \frac{\dot{\mathrm{F}}}{\zeta} \mathrm{d} \zeta=0$ (propriété de l'équation A.2)

(A.5) $\int_{0}^{1} \frac{F}{\zeta} d \zeta=1 \quad(A .4+A .3)$

(A.6) $\dot{F}(\zeta, 0)=-\zeta^{n}+\frac{\zeta}{n}$ (conséquence de A.3)

(A.7) $\lim _{t \rightarrow \infty} F(\zeta, t)=\frac{1}{n} \zeta^{1 / n}$ (état asymptotique)

\section{Résolution approchée}

Nous allons présenter une méthode de résolution approchée de l'équation (A.2), dont la justification sera la précision du résultat auquel elle a abouti.

- soit $G_{0}$ la solution de l'équation A.2, sans la partie intégrale, et vérifiant A.3. On a :

$$
\begin{aligned}
G_{0} & =\zeta \Psi \quad \text { avec }: \\
\Psi(\zeta, t) & =\left(1+(n-1) \zeta^{n-1} t\right)^{\frac{1}{1-n}}
\end{aligned}
$$

$\Psi$ vérifie :

$$
\dot{\Psi}=-\zeta^{n-1} \Psi^{n} .
$$

- On peut chercher une première approximation de $F$, en remplaçant celle-ci par $G_{0}$ dans le second membre de A.2:

$$
\dot{G}_{1}(\zeta, t)=-G_{0}^{n}+\zeta \int_{0}^{1} \frac{G_{0}^{n}}{\zeta} d \zeta
$$

soit :

(A.11)

$$
G_{1}(\zeta, t)=\zeta\left[1+\Psi-\int_{0}^{1} \Psi d \zeta\right]
$$

En effet, $G_{1}$ vérifie l'ensemble des relations (A.3) à (A.6). Elle constitue un développement limité d'ordre 1 de la fonction $\mathrm{F}$ au voisinage de $\mathrm{t}=0$, dans le sens où (A.3) et (A.6) sont vérifiées.

Nous intégrons le terme intégral de cette expression d'une manière approchée, en passant par sa dérivée par rapport au temps. 
Soit :

$$
I=\int_{0}^{1} \Psi(\zeta, t) d \zeta
$$

$$
\frac{\partial \mathrm{I}}{\partial \mathrm{t}}=\int_{0}^{1} \Psi(\zeta, \mathrm{t}) \mathrm{d} \zeta=-\int_{0}^{1} \zeta^{\mathrm{n}-1} \Psi^{\mathrm{n}}(\zeta, \mathrm{t}) \mathrm{d} \zeta
$$

en intégrant par parties:

$$
\begin{aligned}
& \frac{\partial \mathrm{l}}{\partial \mathrm{t}}=-\left[\frac{1}{\mathrm{n}} \zeta^{\mathrm{n}} \Psi^{\mathrm{n}}\right]_{0}^{1} \\
& +\frac{1}{\mathrm{n}} \int_{0}^{1} \zeta^{\mathrm{n}} \frac{\partial}{\partial \zeta} \Psi^{\mathrm{n}} \mathrm{d} \zeta
\end{aligned}
$$

or $\partial / \partial \zeta \Psi^{n}=-n(n-1) \zeta^{n-2} \Psi^{2 n-1} t$, est du premier ordre en $t$ et son intégration par rapport au temps donnera une contribution aux termes du second degré en $t$ dans l'expression de I. On peut donc le négliger puisqu'on ne cherche qu'un développement limité de premier ordre en temps.

On trouve alors:

(A.12) $\frac{\partial \mathrm{I}}{\partial \mathrm{t}} \simeq-\left[\frac{1}{\mathrm{n}} \zeta^{\mathrm{n}} \Psi^{n}\right]_{0}^{1}=-\frac{1}{\mathrm{n}} \Psi_{1}^{\mathrm{n}}$

où :

(A.13) $\Psi_{1}(\mathrm{t}) \equiv \Psi(1, \mathrm{t})=(1+(\mathrm{n}-1) \mathrm{t})^{\frac{1}{1-n}}$

$\Psi_{1}$ vérifie : $\quad \Psi_{1}(\mathrm{t})=-\Psi_{1}^{n}$

ce qui permet d'intégrer (A.12) par rapport au temps :

$$
1 \simeq c+\frac{\Psi_{1}}{n}
$$

Nous déterminons $\mathrm{c}$ pour que au temps $\mathrm{t}=0$ on ait une égalité. On trouve finalement:

(A.14)

$$
I=\int_{0}^{1} \Psi(\rho, t) d \rho \simeq 1-\left[\frac{1-\Psi_{1}(t)}{n}\right]
$$

On en déduit :

$$
G_{1} \simeq \rho\left[\Psi(\rho, t)+\frac{1-\Psi_{1}(t)}{n}\right]
$$

On voit que $G_{1}$ sous la forme (A.15) ne vérifie plus (A.5) que jusqu'au premier ordre en $t$, mais qu'il vérifie par contre la condition A.7 :

$$
\lim _{t \rightarrow \infty} G_{1}(\zeta, t)=\frac{\zeta}{n}
$$

C'est-à-dire qu'au moins en $\zeta=1$ (à la paroi de la galerie), $\mathrm{G}_{1}$ a la bonne limite à l'infini :

$$
\lim _{t \rightarrow \infty} G_{1}(1, t)=\frac{1}{n}=\lim _{t \rightarrow \infty} F(1, t)
$$

Déviateur à la paroi :

Ceci permet de proposer au moins une fonction d'évolution du déviateur à la paroi de la galerie :

$$
F(1, t) \simeq \frac{1+(n-1) \Psi_{1}(t)}{n}
$$

soit en désignant ce déviateur en paroi par la fonction $S_{n}(t)$, plus explicitement :

$$
S_{n}(t)=\frac{1}{n}+\frac{n-1}{n}(1+(n-1) t)^{\frac{1}{1-n}}
$$

La figure 5 permet de comparer la courbe de cette fonction avec celle obtenue numériquement pour le déviateur à la paroi dans le cas $n=3$. On voit que $\mathrm{S}_{\mathrm{n}}$ suit avec une très grande précision la courbe numérique. L'accord entre les deux courbes reste excellent, dans la plage des valeurs usuelles de $n$ ( $\mathrm{n}=1$ à 5 ).

\section{Déviateur dans le massif:}

Nous reprenons la fonction $G_{1}$ donnée par (A.15) et nous la modifions légèrement pour corriger sa limite à l'infini pour $\zeta \neq 1$. Nous posons :

$$
\mathrm{S}_{\mathrm{n}}(\zeta, \mathrm{t}) \equiv \zeta \Psi(\zeta, \mathrm{t})+\frac{\zeta^{\frac{1}{n}}}{\mathrm{n}}(1-\Phi(\zeta, \mathrm{t}))
$$

où :

(A.20)

$\Phi(\zeta, t) \equiv \Psi\left(\zeta^{\frac{1}{n}}, t\right)=\left(1+(n-1) \zeta^{\frac{n-1}{n}} t\right)^{\frac{1}{1-n}}$

on voit que $\Phi$ vérifie :

$$
\dot{\Phi}(\zeta, t)=-\zeta^{\frac{n-1}{n}} \Phi^{n}(\zeta, t)
$$

et que $S_{n}(\zeta, t)$ vérifie les relations (A.3). (A.6) et (A.7) que doit vérifier $F(\zeta, t)$, et qu'elle vérifie (A.5) jusqu'au premier ordre (en raison de (A.3) et (A.6)). On peut encore montrer, à laide de (A.22) que :

$$
\lim _{\mathrm{n}-1} \mathrm{~S}_{\mathrm{n}}(\zeta, \mathrm{t})=\zeta
$$

c'est-à-dire que $\mathrm{S}_{\mathrm{n}(\mathrm{s}, \mathrm{t})}$ tend vers la solution exacte du problème $n-1$.

Pour $\zeta=1, S_{n}(\zeta, t)$ coïncide avec $S_{n}(t)$ dont nous nous sommes convaincus de la précision; de plus, elle possède la bonne limite pour tout $\zeta$. Nous pouvons donc penser que, sous réserve d'une comparaison avec des exemples numériques, $\mathrm{S}_{\mathrm{n}}(\zeta, t)$ est une très bonne approximation de $\mathrm{F}(\zeta, \mathrm{t})$. 